Fermilab-TM-2737-AD May 14, 2020

\title{
Energy deposition and radiological studies with MARS15 code for NuMI-AIP project
}

\author{
Igor Rakhno
}

\section{Introduction}

The studies described below have been performed for NuMI Accelerator Improvement Project (NuMI AIP). This work has been done to extend previous studies [1] and address new issues encountered as the project evolves. The calculations have been performed for both normal operation and an accident scenario.

This report deals with the following calculation results obtained with MARS15 code [2]:

(i) prompt dose distributions around an air diverter placed above the first horn;

(ii) energy deposition distributions in the beryllium window downstream of the target;

(iii) energy deposition distributions in the decay pipe upstream window with a repair patch applied;

(iv) energy deposition distributions in the decay pipe.

\section{Prompt dose distributions around the air diverter}

The air diverter has been introduced in order to deal with potential extra heating of the stripline downstream of the first horn at $1 \mathrm{MW}$ beam power and corresponding enhanced stripline vibrations. From the standpoint of radiation shielding, the air diverter represents a (three-legged) penetration in the shielding T-block right above the stripline (see Fig. 1).

For calculations performed for normal operations, intensity of the incoming $120-\mathrm{GeV}$ proton beam is assumed to be $1.3 \times 10^{13}$ proton/second which corresponds to $250 \mathrm{~kW}$ and simplifies comparisons with various dose distributions calculated previously at earlier stages of NuMI project. For operations at $1 \mathrm{MW}$, a simple scaling with a factor of 4 can be performed. Time interval between pulses is assumed to be 1.33 second. The beam is assumed to have a Gaussian distribution in both horizontal and vertical plane, with $\sigma$ being equal to $1.5 \mathrm{~mm}$ in each plane. Angular distribution of the incoming beam is also assumed to be a Gaussian, with $\sigma$ being equal to 0.09 mradian in each plane. 

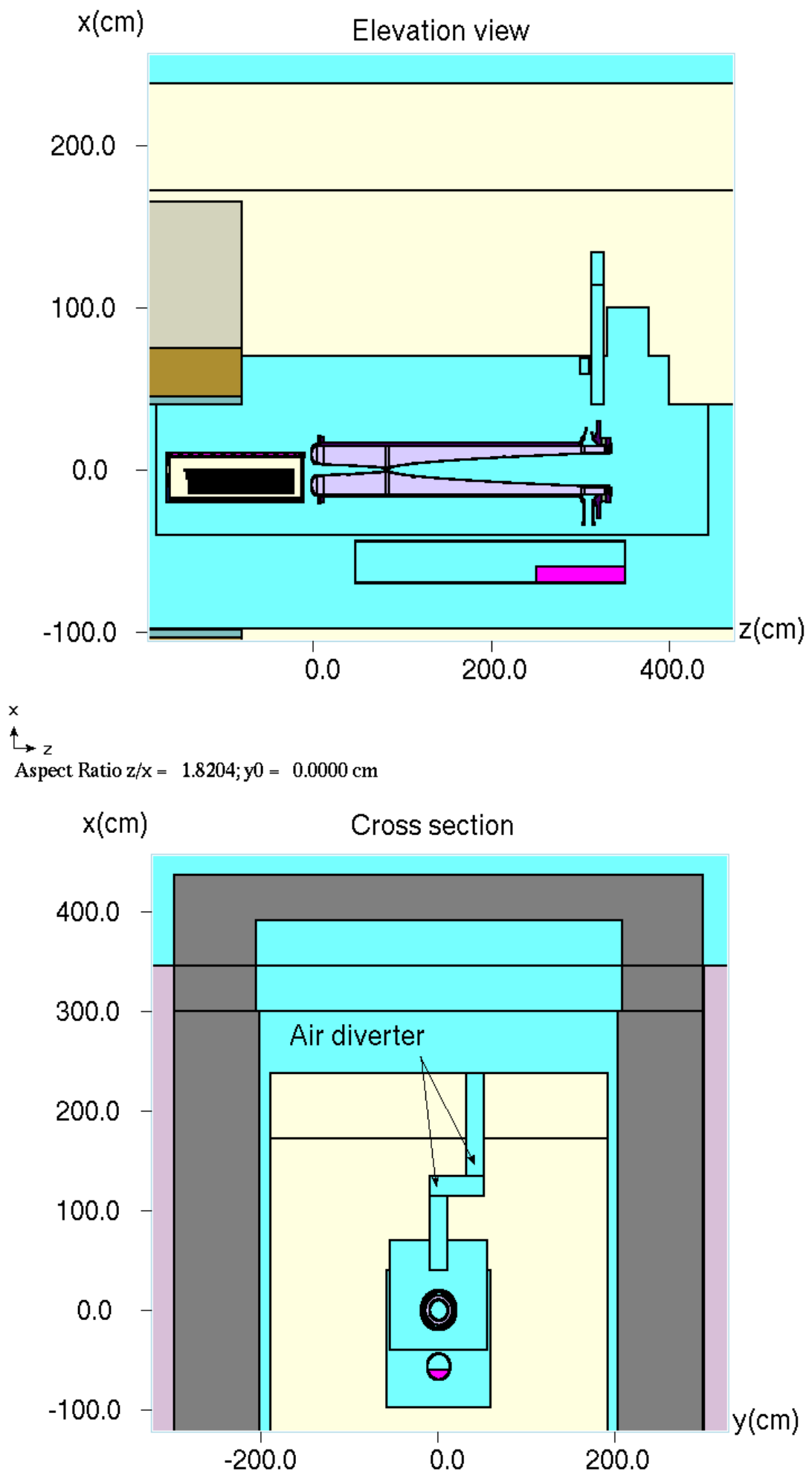

$\stackrel{\mathbf{x}}{\leftrightarrow}$

$\mathrm{x}: \mathrm{y}=1: \quad 1.1263 ; \mathrm{z} 0=316.0000 \mathrm{~cm}$

Fig. 1. Elevation view (top) and cross section (bottom) of the MARS model that show the air diverter above the first horn and stripline. 
For the accident scenario, one assumes that the beam is displaced $6 \mathrm{~mm}$ off center and hits four fins with wings shown in Fig. 2. Such a scenario represents a credible worst case from the standpoint of peak energy deposition in the hadron absorbed core.

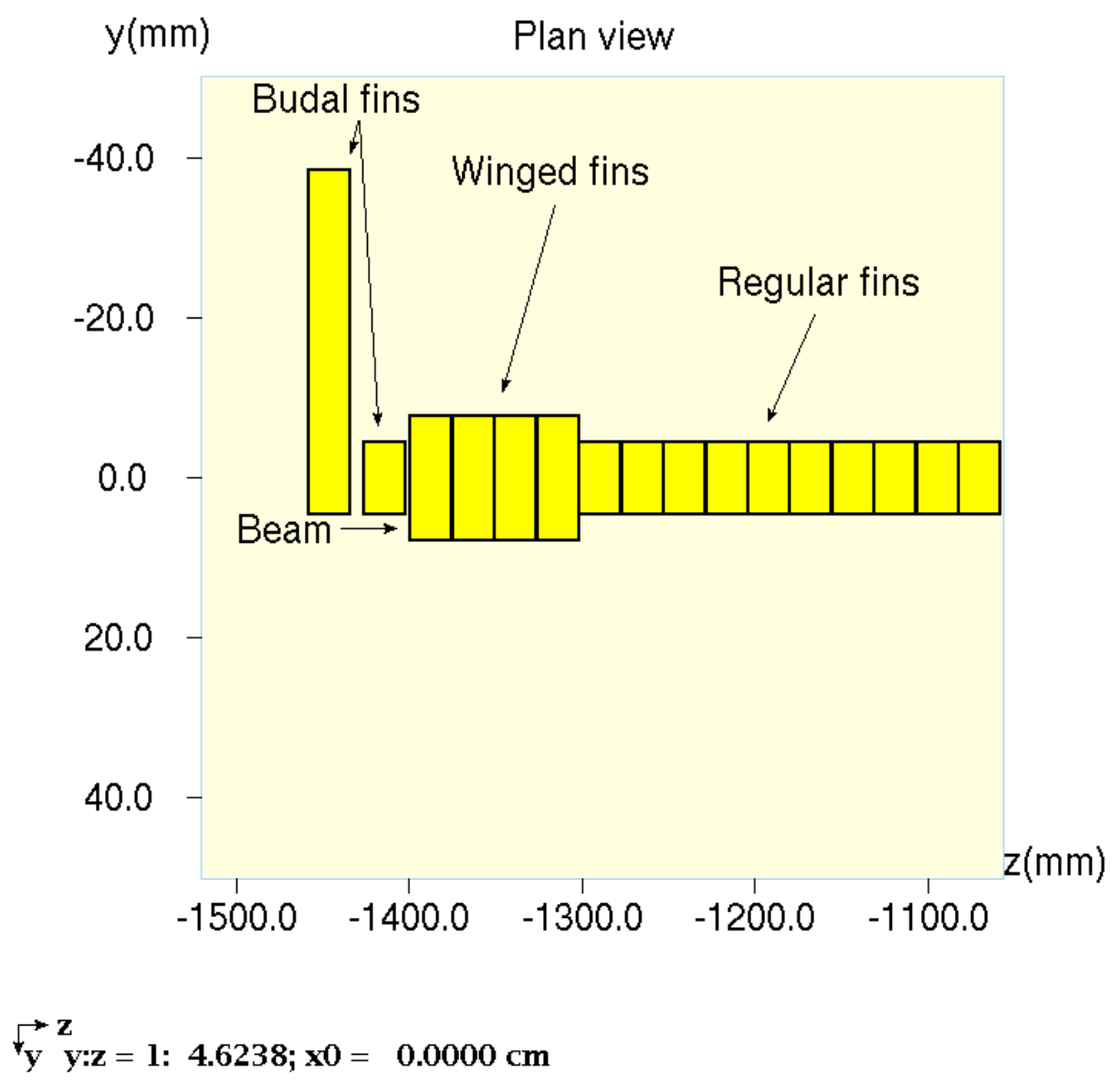

Fig. 2. Fragment of the MARS model of the target that shows location of the displaced beam at the accident.

Also, a separate comparison study has been performed to see if a more detailed description of the spatial and angular distribution of the incoming beam makes any essential difference (see Sec. 5). Namely, the correlations that exist between spatial and angular coordinates of particles in the incoming beam have been taken into account. It has been done by means of a dedicated modeling procedure developed previously [3] and $\alpha$ - and $\beta$-functions calculated for the beamline [4].

The calculated prompt dose distributions around the air diverter are shown in Figs. 3 and 4 for normal operation and the accident, respectively. One can see that in the target chase the accident represents a less severe danger-compared to normal operation- from the standpoint of radiation safety. It is an expected outcome because for the accident scenario in the target chase the misbehaved beam interacts mostly with four winged fins, and the major beam interactions with matter occur well downstream of the target chase. 


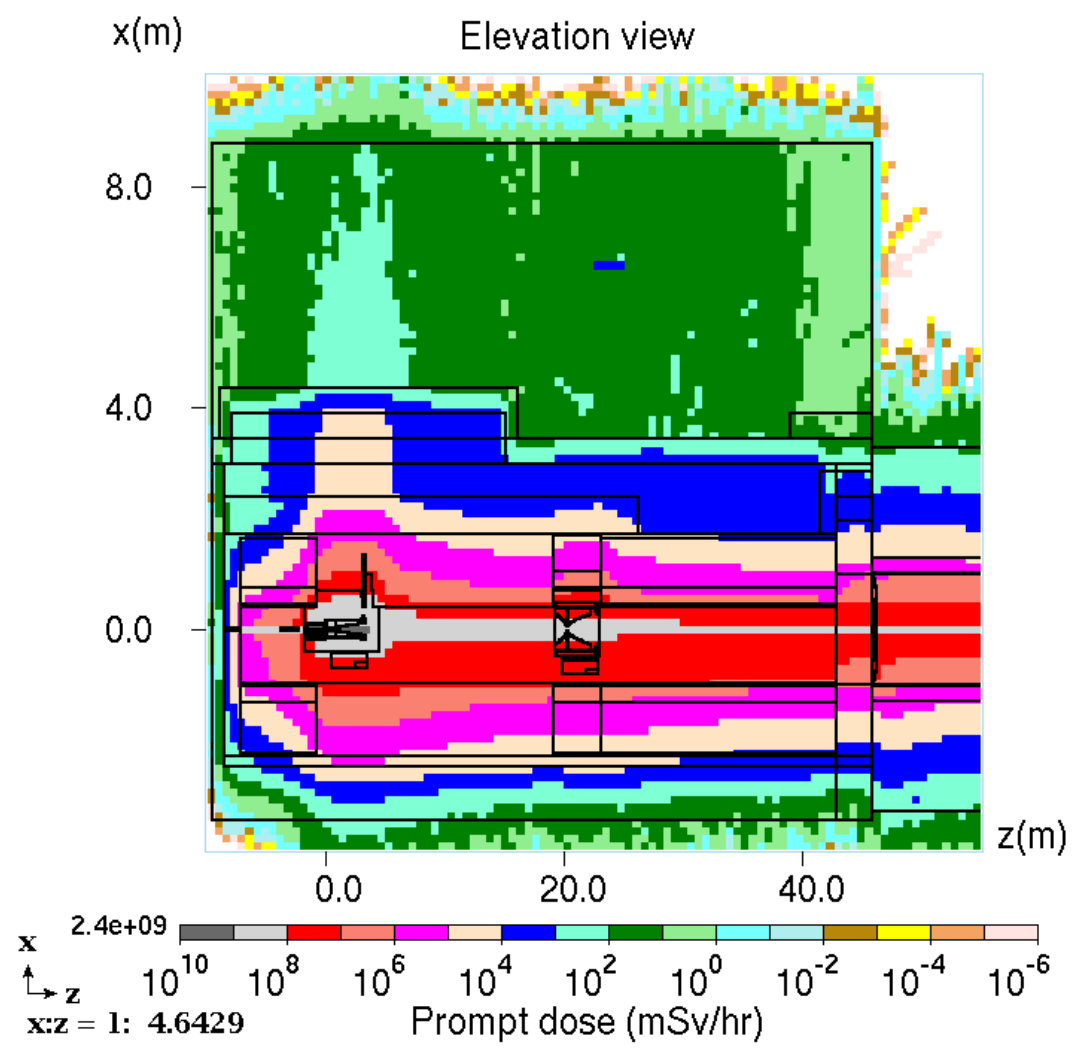

$x(m)$ Cross section through the air diverter $(Z=3.2 \mathrm{~m})$
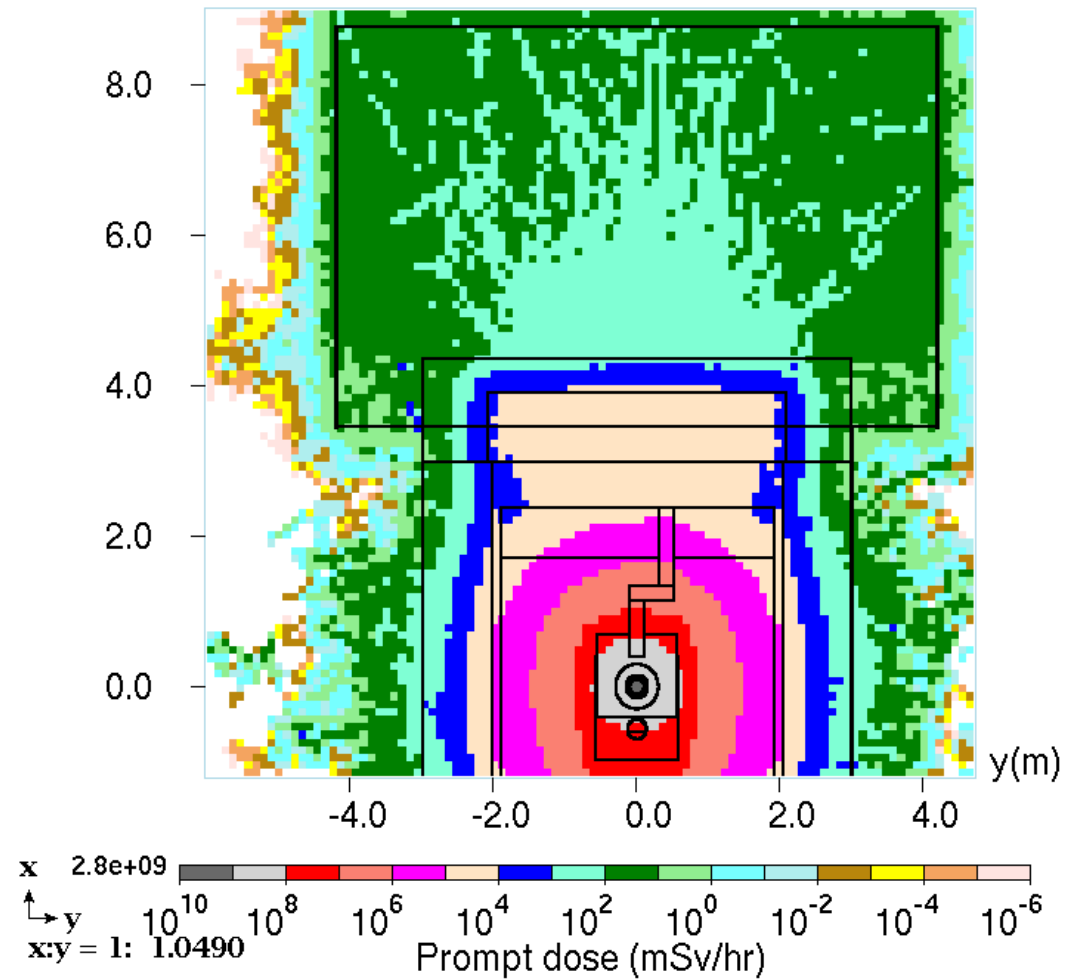

Fig. 3. Calculated distributions of prompt dose around the target chase for normal operations. Power of the incoming beam is assumed to be $250 \mathrm{~kW}$. 

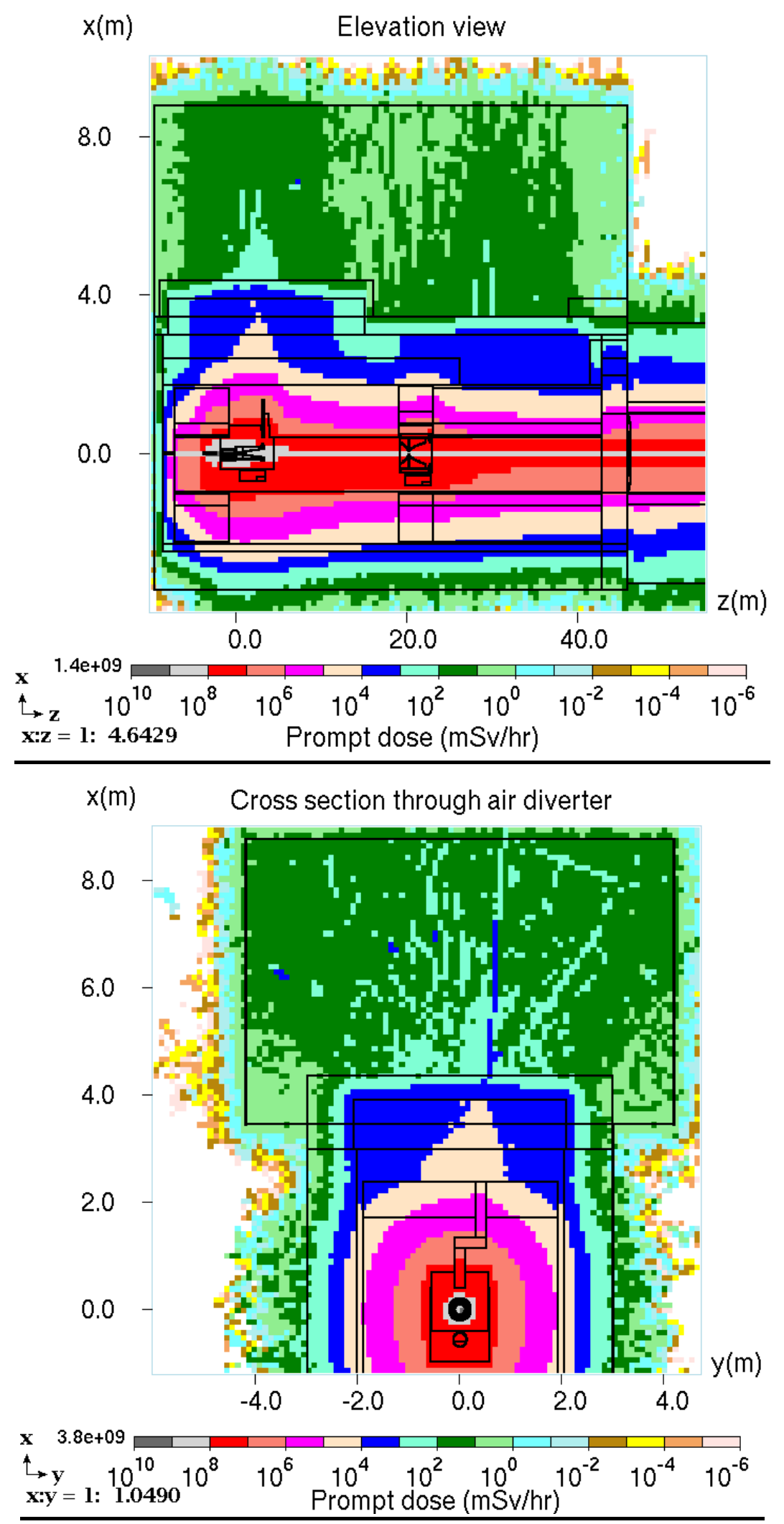

Fig. 4. Calculated distributions of prompt dose around the target chase for the accident scenario. Power of the incoming beam is assumed to be $250 \mathrm{~kW}$. 


\section{Energy deposition in the beryllium window downstream of the target}

The beryllium window downstream of the target is re-examined because thermal analysis based on initial energy deposition calculations has shown that this window can be overheated during operations at beam power of $1 \mathrm{MW}$. An additional cooling omega loop, shown in Fig. 5, has been introduced in order to mitigate the overheating issue [5]. This cooling loop (filled with water) has been implemented in our MARS model (see Figs. 6 thru 8) and calculations of energy deposition in the window have been performed.

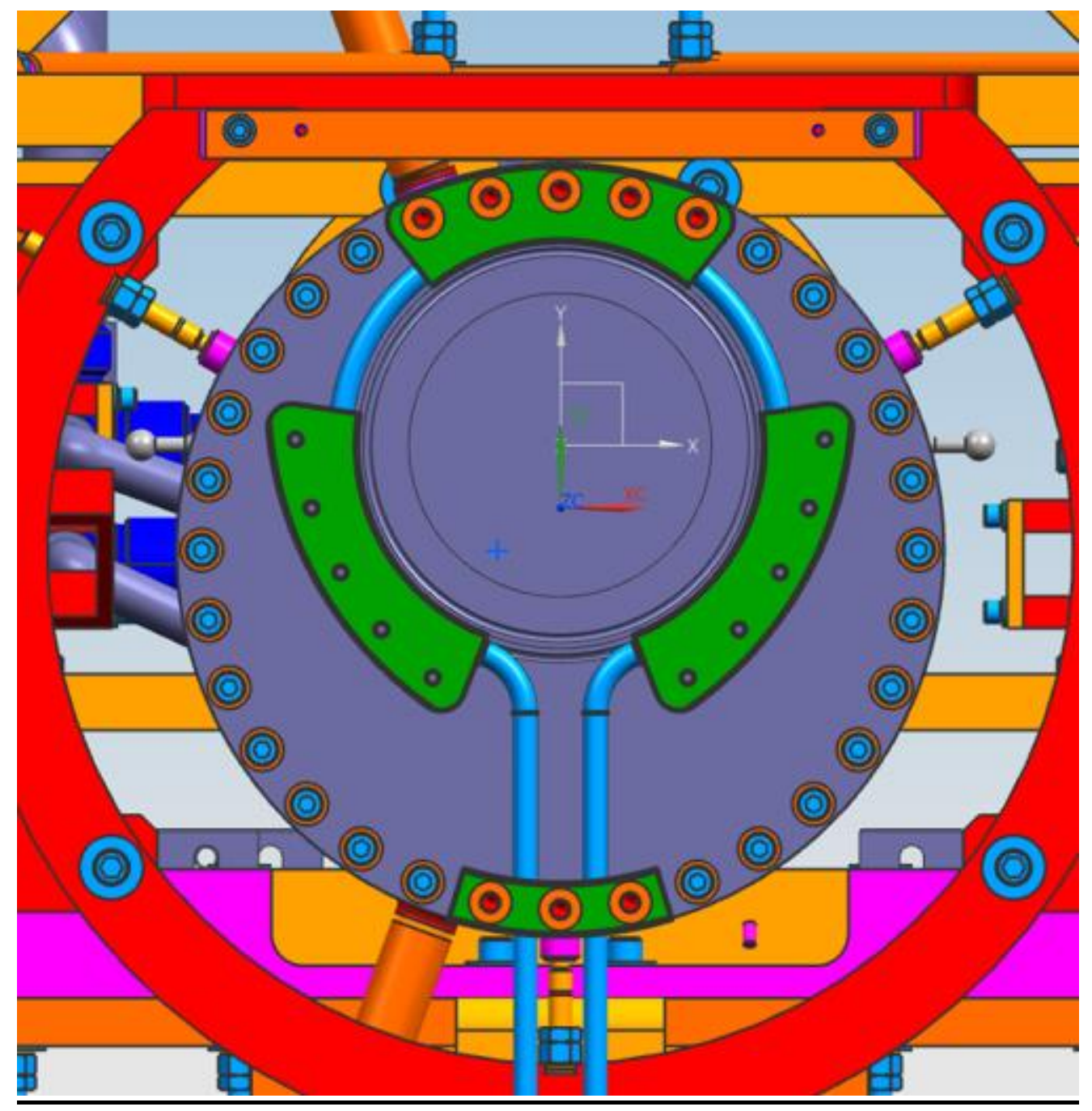

Fig. 5. A cooling $\Omega$-shaped loop (blue color) attached to the target downstream beryllium window.

For normal operations, the calculated energy deposition (ED) is provided in two files with names "ed_in_target_DS_Be_window_...txt" which are self-explanatory. The units of ED are the same that were used previously, $\mathrm{GeV} /\left(\mathrm{cm}^{3 *}\right.$ proton). Three columns were added to the file with ED in the Be window itself with names DPA1, DPA2 and DPA3 (units are dpa/proton). The three columns correspond to three different DPA models used in MARS15. The Be window has $\mathrm{R}=6.75$ $\mathrm{cm}$. 67 radial bins were used in calculations for normal operations, so that the bin size is $67.5 \mathrm{~mm}$ / $67=1.00746 \mathrm{~mm}$. 

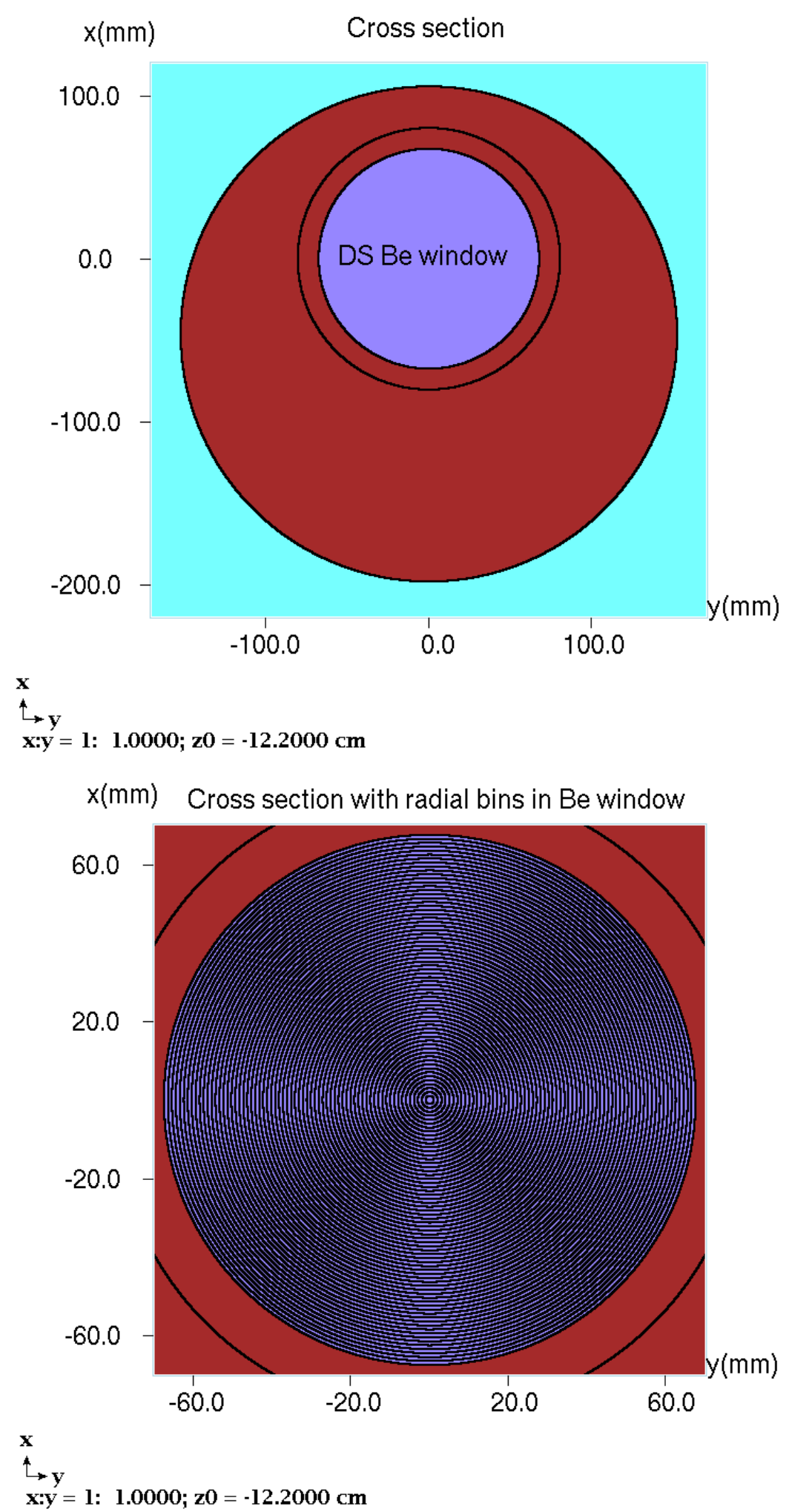

Fig. 6. A cross section of the model taken thru the target DS beryllium window (top) and a fragment that shows the radial bins used for scoring energy deposition in the window (bottom). Dark brown, violet and pink colors in Figs. 6-8 correspond to aluminum, beryllium and water, respectively. 


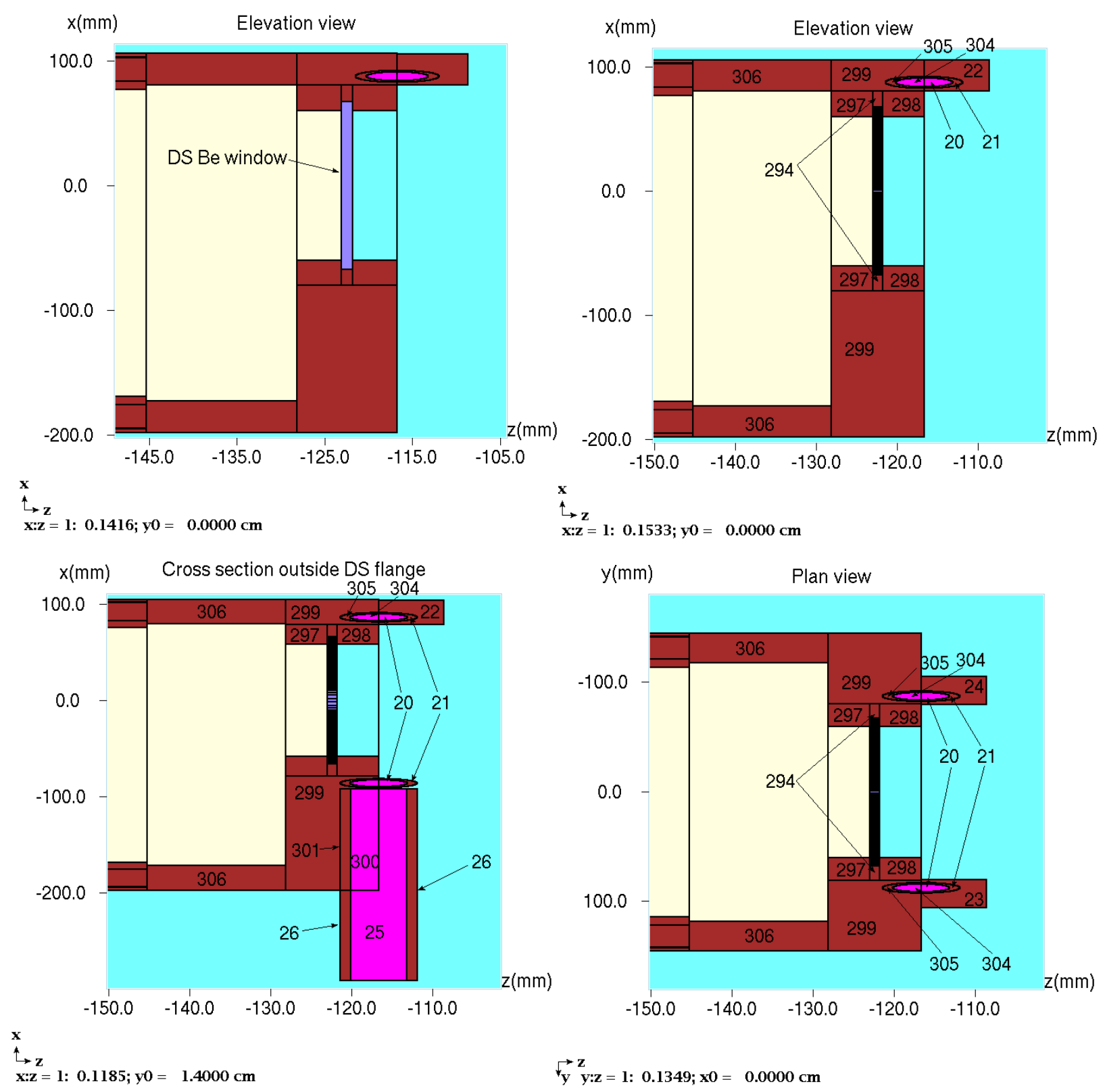

Fig. 7. An elevation view of the downstream part of the target assembly with the beryllium window taken at $\mathrm{Y}=0$ with and without radial bins (top right and left, respectively), and at $\mathrm{Y}=1.4 \mathrm{~cm}$ (bottom, left). Also shown is the plan view of the downstream part of the assembly (bottom, right) taken at $X=0$. The numerical labels on the figures correspond to the region numbers used in the above-mentioned files with calculated energy deposition, ed_in_target_DS_Be_window_...txt. Dark brown, violet and pink colors in Figs. 6-8 correspond to aluminum, beryllium and water, respectively. 


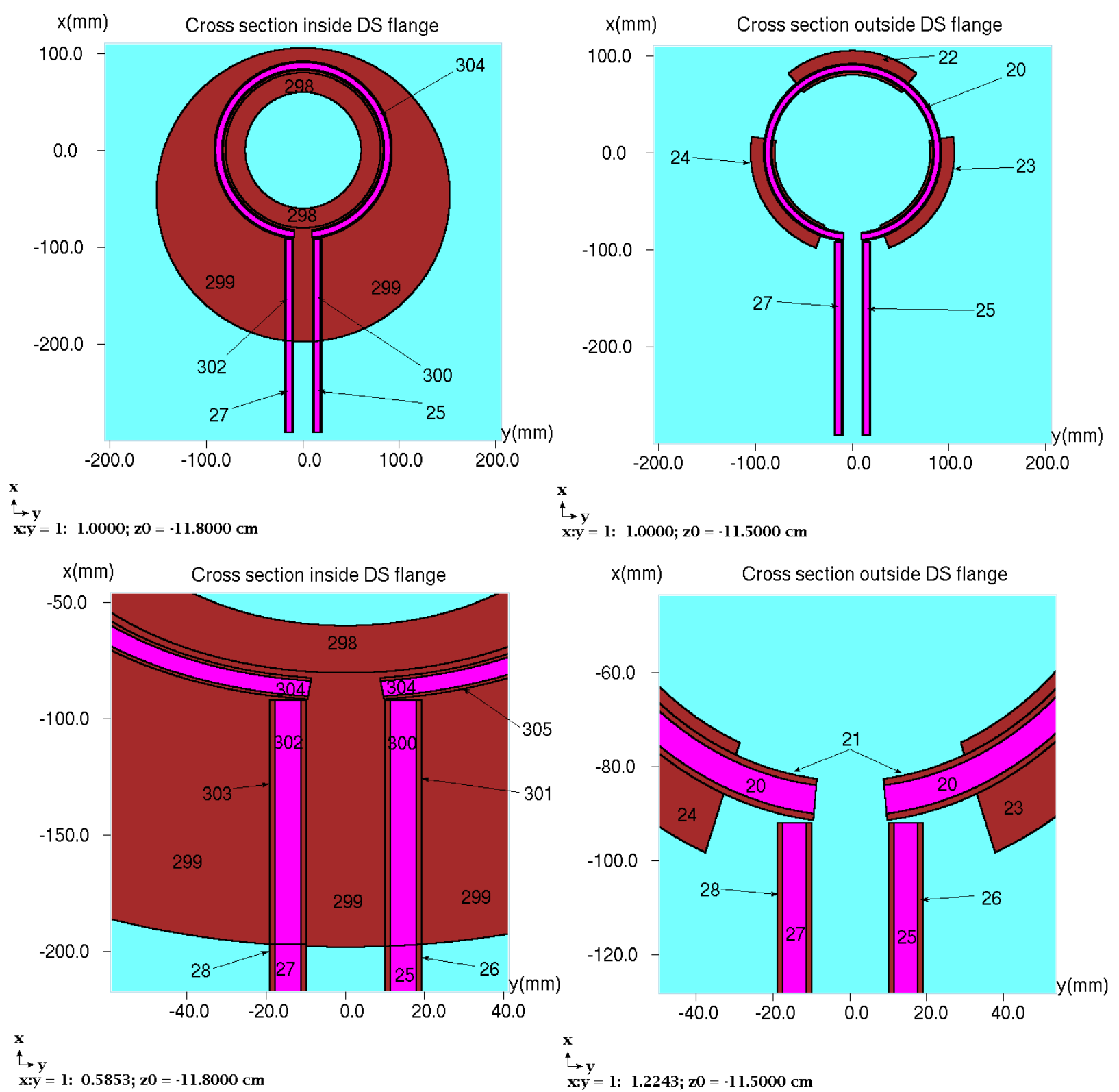

Fig. 8. Cross sections taken inside (left top) and outside (right top) of the DS target assembly flange. Fragments of the cross sections are shown in corresponding bottom parts of the Figure. The numerical labels on the figures correspond to the region numbers used in the above-mentioned files with calculated energy deposition, ed_in_target_DS_Be_window_...txt. Dark brown, violet and pink colors in Figs. 6-8 correspond to aluminum, beryllium and water, respectively.

For the accident scenario, the 6-mm offset has been applied to the model when building the radial bins in the window (see Fig. 9). Detailed distribution of the calculated energy deposition in the window is given in the following files:

- ed_in_target_DS_Be_window_Accident_6mm_off-center_dR_about_0p5mm.txt;

- ed_in_target_DS_Be_window_flanges_and_water_pipes_Accident_6mm_off.txt. 


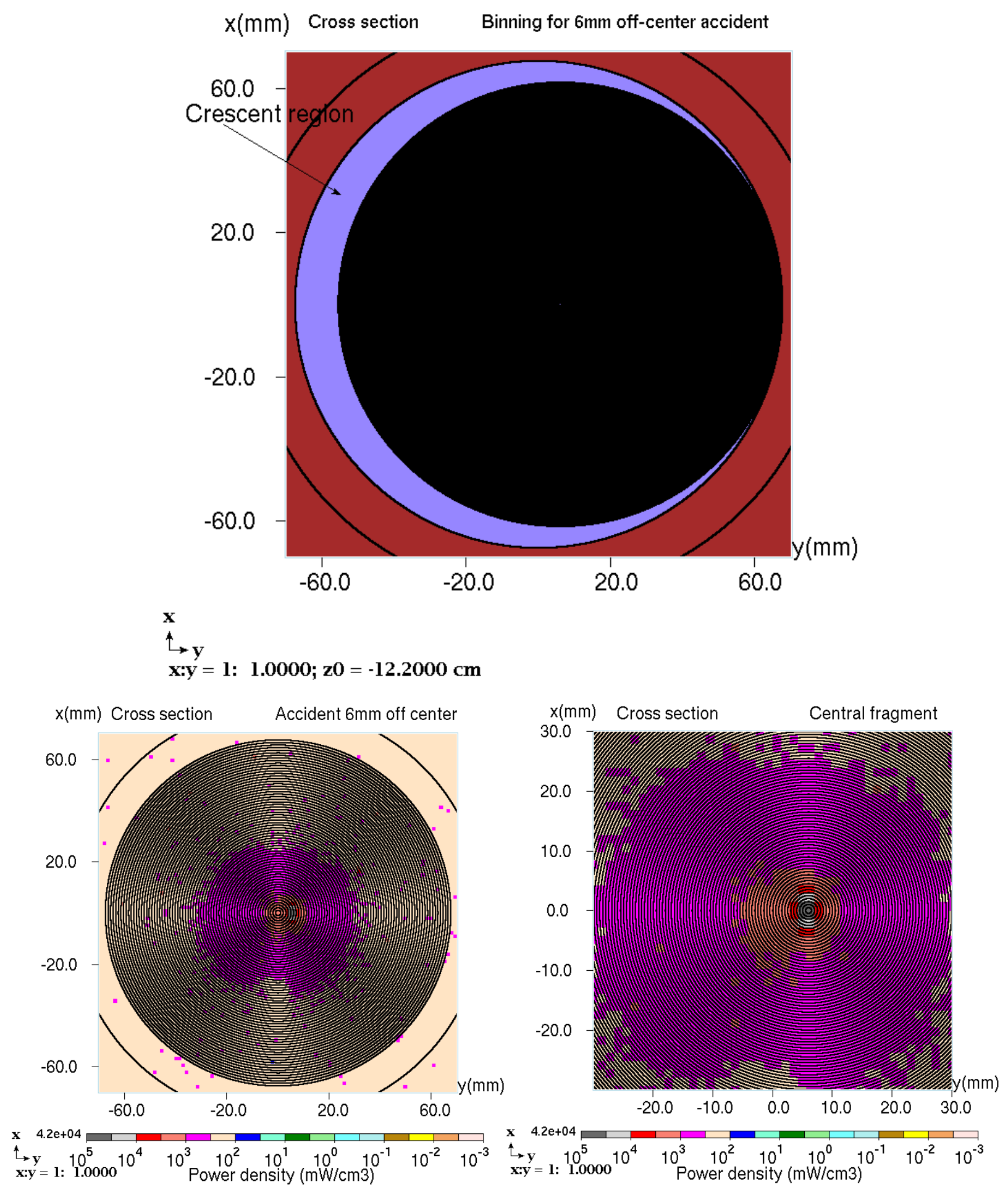

Fig. 9. A cross section of the model of the window (top) that shows the asymmetric radial binning used in the model. The black area represents the large number of small radial bins $(\Delta \mathrm{R}=0.5 \mathrm{~mm})$ that can't be shown properly with a limited computer screen resolution. Also shown is a sample of calculated energy deposition in the window with symmetric (bottom left) and asymmetric (bottom right) radial binning in the model with $\Delta \mathrm{R}=1 \mathrm{~mm}$. For normalization purpose, power of the incoming beam is assumed to be $250 \mathrm{~kW}$. 


\section{Energy deposition in the decay pipe upstream window repair patch}

The decay pipe upstream aluminum window will be operating at harsh radiation environment, and one of possible failure modes for such a window is appearance of cracks. In order to develop a fast and reliable repair scenario, an application of a repair patch has been suggested (plan B) using the same material, that is aluminum (see Fig. 10). For the accident scenario, an asymmetric radial binning has been used in the model (see Fig. 11). Energy deposition calculations have been performed for such an assembly with a repair patch.
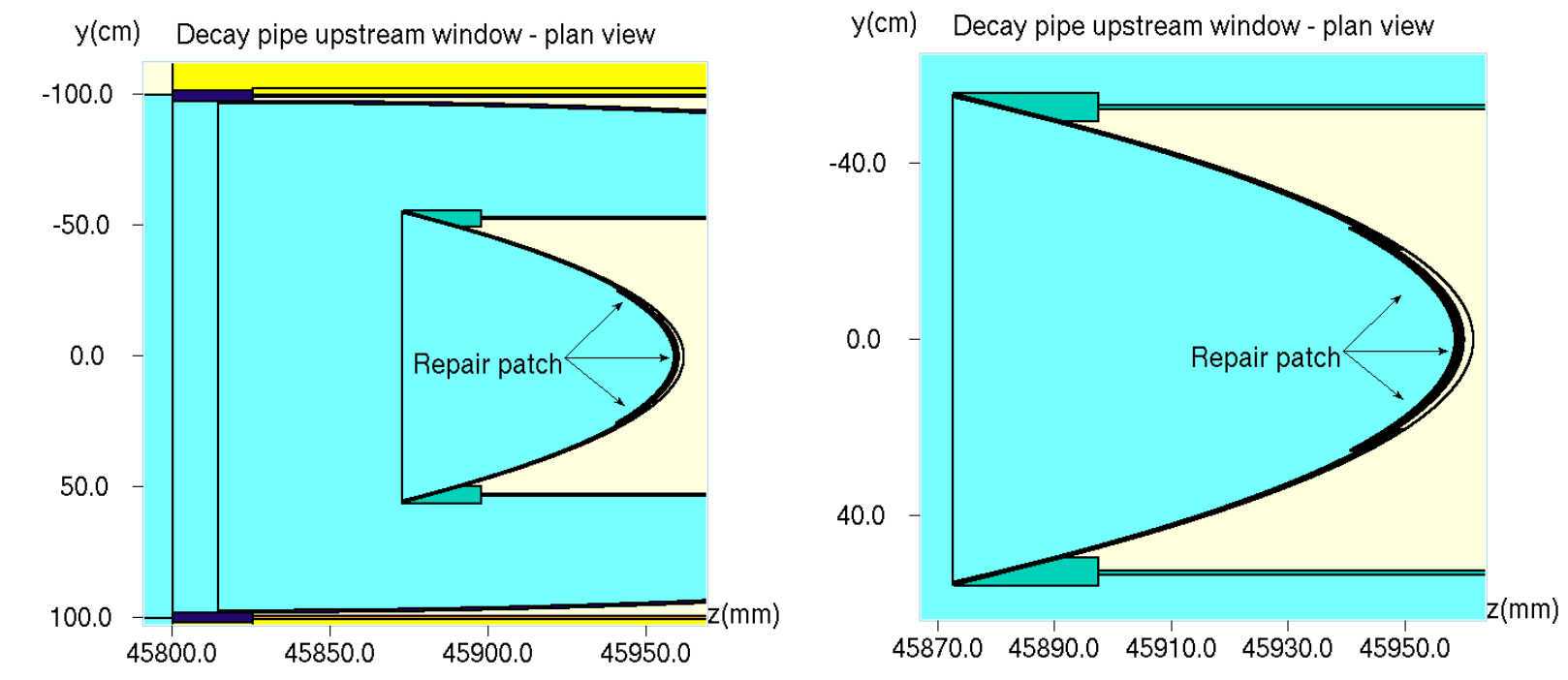

$\vec{\gamma}_{\mathrm{y}}^{\mathrm{z}} \mathrm{y}: \mathrm{z}=1: 0.0829 ; \mathrm{x} 0=0.0000 \mathrm{~cm}$

$\vec{\gamma}_{\mathrm{y}} \mathrm{z}: \mathrm{y}=1: 0.0754 ; \mathrm{x} 0=0.0000 \mathrm{~cm}$

$y(\mathrm{~mm})$ Decay pipe upstream window - plan view
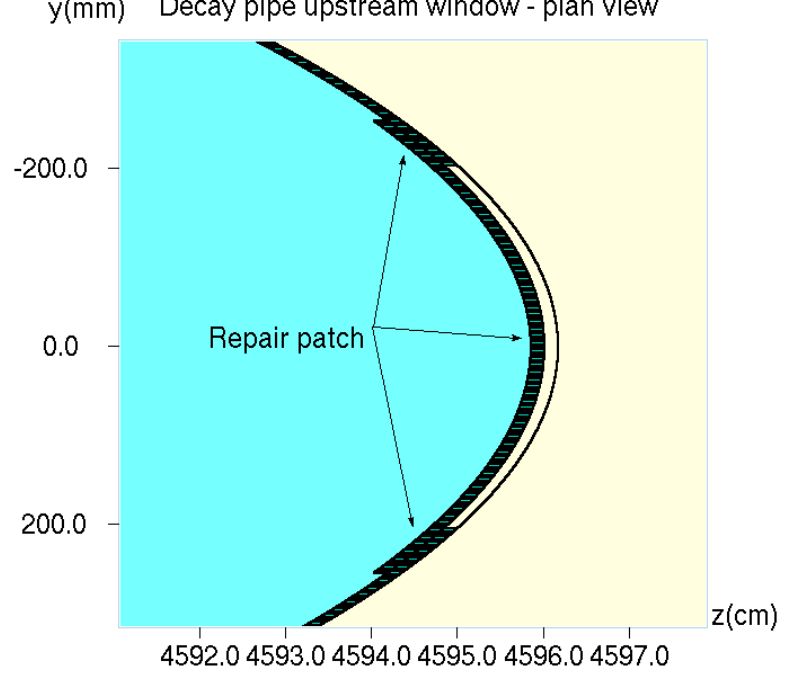

$y(\mathrm{~mm})$ Decay pipe upstream window - plan view (fragment)

$\overrightarrow{\vec{y}_{\mathrm{y}}} \mathrm{y}: \mathrm{z}=1: 0.1037 ; \mathrm{x0}=0.0000 \mathrm{~cm}$

$\vec{y}_{y: z=1: 0.1140 ; x 0}^{z}=0.0000 \mathrm{~cm}$

Fig. 10. Various views of the decay pipe upstream window with a repair patch applied. 


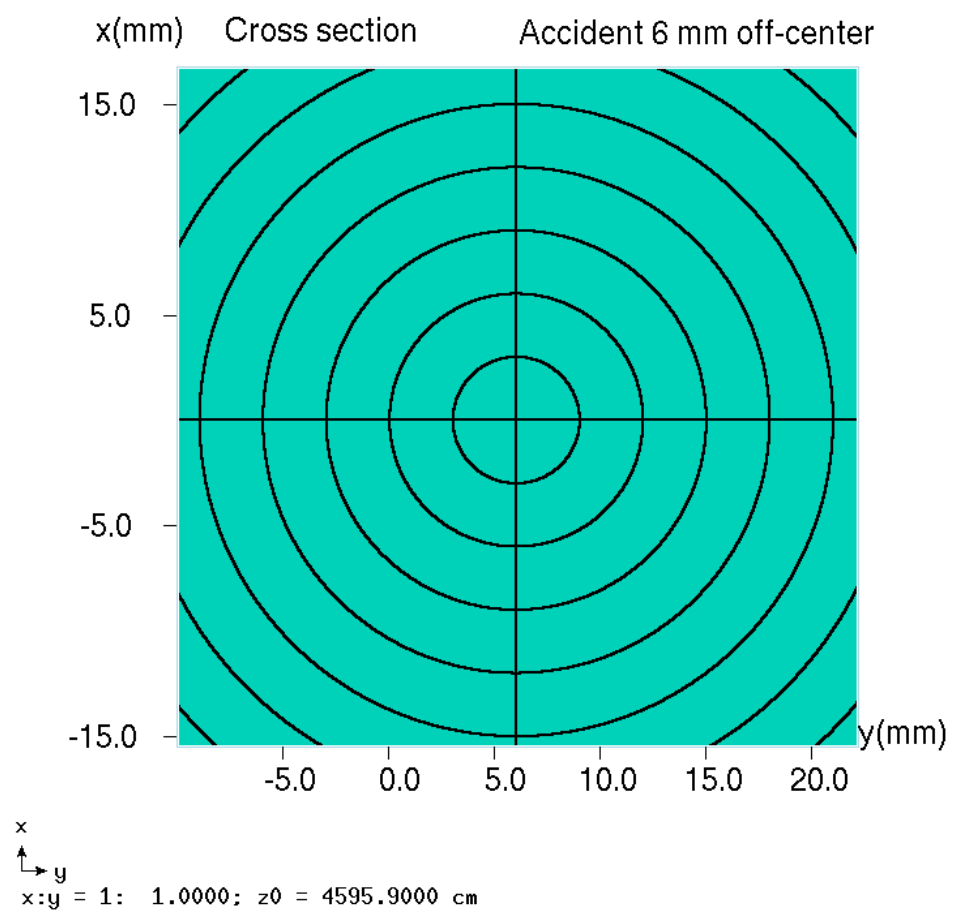

Fig. 11. An asymmetric radial binning used in the model for the accident scenario with the 6-mm radial beam offset.

The calculated energy deposition distributions for the window assembly are given in the following files:

- ed_in_dkp_us_al_window_normal_ops.txt;

- ed_in_dkp_us_al_window_accident_6mm_off.txt;

- ed_in_repair_patch_of_dkp_us_al_window_normal_ops.txt;

- ed_in_repair_patch_of_dkp_us_al_window_accident_6mm_off.txt.

Energy deposition data for every single item (support ring, radial bin of semi-elliptical head or window etc) are given in four columns which correspond to 4 azimuthal bins 90 degrees each (see Fig. 12). The correspondence between the columns and the bins is shown in the scheme below. When we are looking at the scheme, it means we are looking along the beam (downstream).

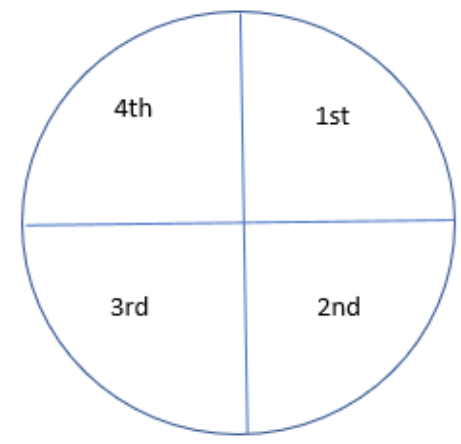

Fig. 12. Four bins in azimuth used for scoring the calculated energy deposition. 
The different regions of the assembly (various rings and the semi-elliptical head) are numbered according to Fig. 13 below. The regions 2 thru 5 are filled with Aluminum, the very thin region 6 - Titanium, and regions 1, 7, 8, 9- steel A36 $\left(7.85 \mathrm{~g} / \mathrm{cm}^{3}\right)$. The regions of the semi-elliptical head, labeled h1 thru h9, are also filled with steel A36. The EDEP data for the Aluminum window itself are given in a separate file.

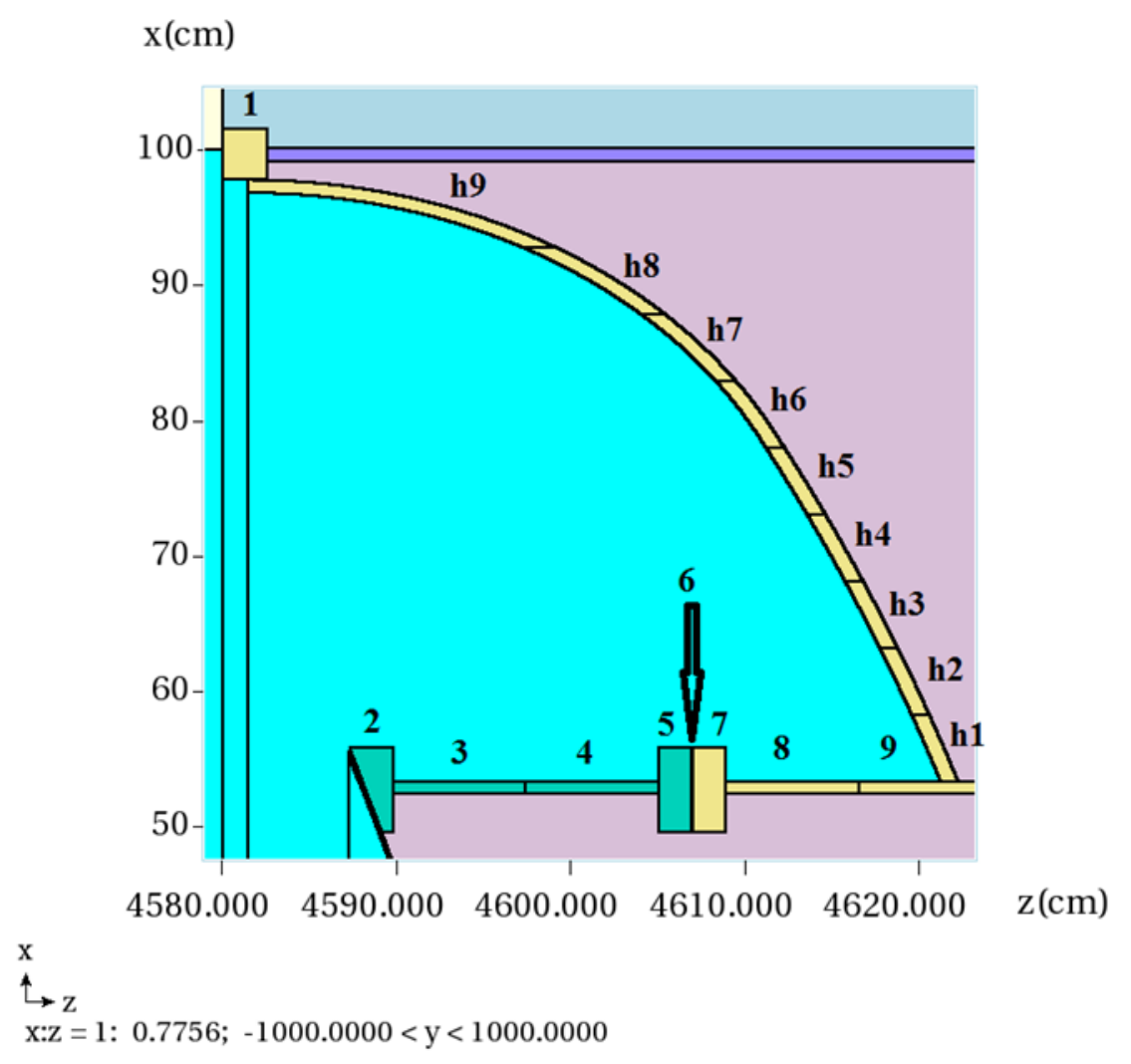

Fig. 13. A fragment of the model of the decay pipe upstream window assembly. The numerical labels show various components of the model with corresponding energy deposition values given in the above-mentioned files.

\section{Energy deposition distribution in the decay pipe}

Thermal analysis performed previously for the decay pipe revealed that overheating of the steel pipe may become an issue at the incoming beam power of $1 \mathrm{MW}$ [6]. Therefore, a detailed distribution of energy deposition in the steel pipe and concrete layers around it has been calculated (for normal operations only) and provided for further thermal analysis. Various views of the MARS model of the decay pipe are shown in Fig. 14. The transition region between the target chase and decay pipe introduces some azimuthal asymmetry to the distribution, mostly in the upstream part of the decay part. The asymmetry makes it necessary to use the binning in azimuth shown in Fig. 14. The asymmetry introduced by the passageway on the right side is almost negligible.

Also, a separate comparison study has been performed to see if a more detailed description of the spatial and angular distribution of the incoming beam makes any essential difference. Namely, the 
correlations that exist between spatial and angular coordinates of particles in the incoming beam have been taken into account. It has been done by means of a dedicated modeling procedure developed previously [3] and $\alpha$ - and $\beta$-functions calculated for the beamline [4].

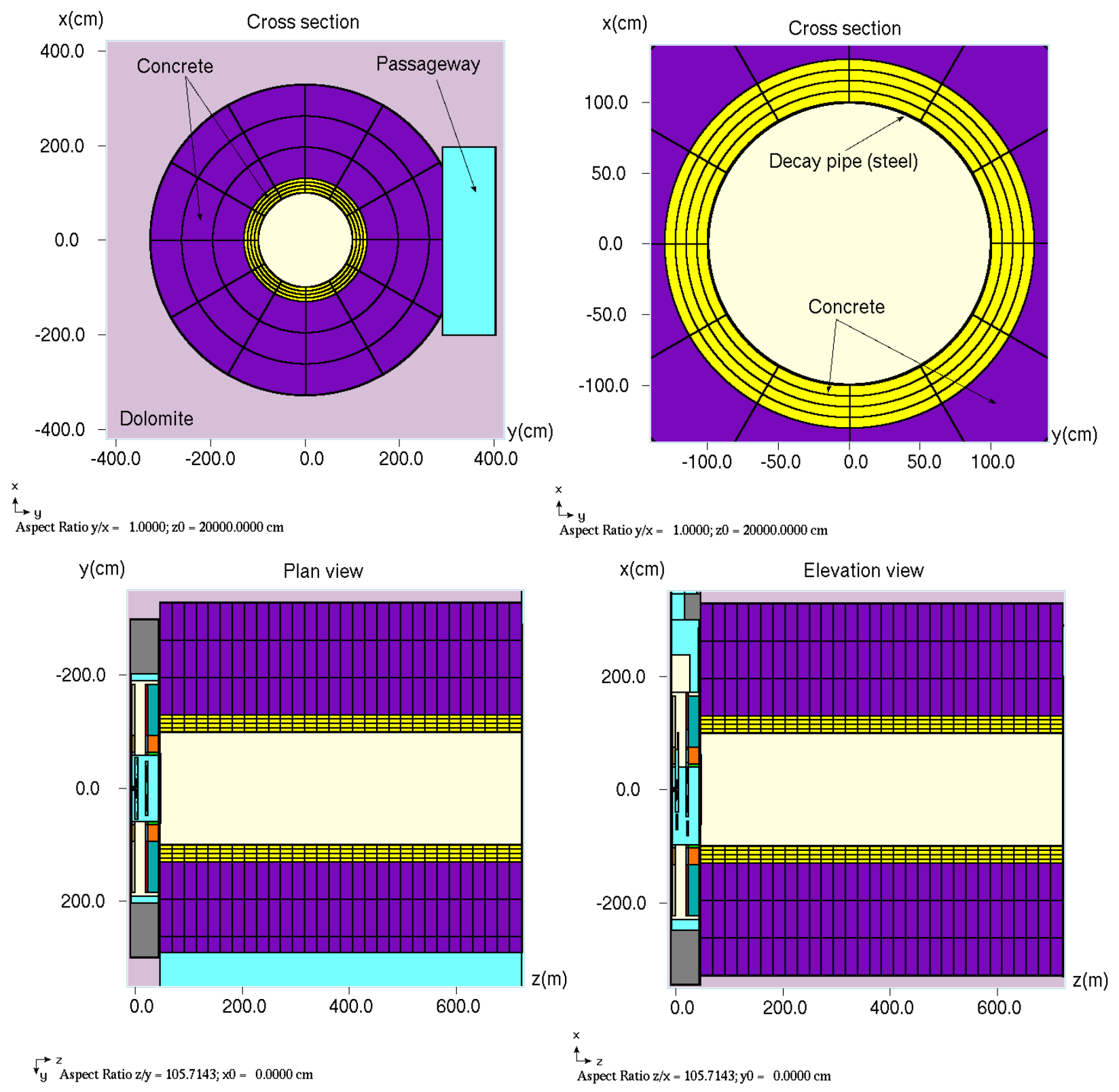

Fig. 14. Cross section of the decay pipe and concrete layers around it (top) as well as plan and elevation views (bottom left and right, respectively).

The right coordinate system used in the model is shown in Fig. 15. The calculated distribution of energy deposition in the decay pipe region (both in steel and concrete layers) is given in the file ed_in_decay_pipe.txt which has self-explanatory column titles including density of material. 
Comparisons between the energy deposition distributions calculated with the approximate algorithm (without correlations between spatial and angular coordinates) and with a refined algorithm (with correlations between spatial and angular coordinates) are shown in Table 1 and Fig. 16 below. One can see that no tangible difference between those two options can be detected. One should mention, however, that from statistical standpoint, the calculations performed without the spatial-angular correlations in the incoming beam are approximately 5 times better than that with the correlations.

All the files with the calculated energy deposition distributions mentioned above are available at [7].

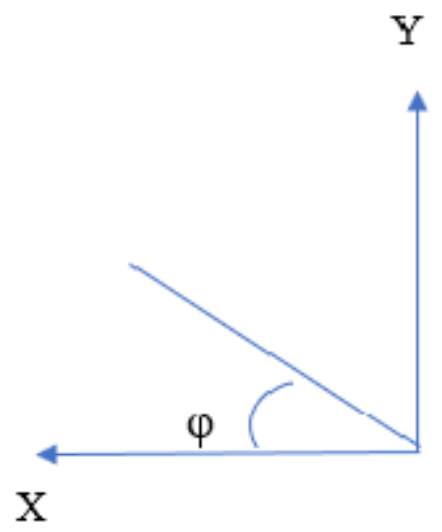

Fig. 15. Definition of the azimuthal angle in the right coordinate system used for the decay pipe model.

Table 1. Energy deposition in a fragment of decay pipe for NuMI-AIP at $Z=250 \mathrm{~m}$ (arbitrary units)

\begin{tabular}{|c|c|c|}
\hline Cell & $\begin{array}{c}\text { Without spatial-angular } \\
\text { correlations in the incoming } \\
\text { beam }\end{array}$ & $\begin{array}{c}\text { With spatial-angular } \\
\text { correlations in the incoming } \\
\text { beam }\end{array}$ \\
\hline 1 -cm thick beam pipe & $4.765 \pm 0.019^{*}$ & $4.689 \pm 0.040$ \\
\hline Adjacent concrete layer & $0.3464 \pm 0.0016$ & $0.3487 \pm 0.0034$ \\
\hline
\end{tabular}

"The statistical uncertainty is $1 \sigma$. 

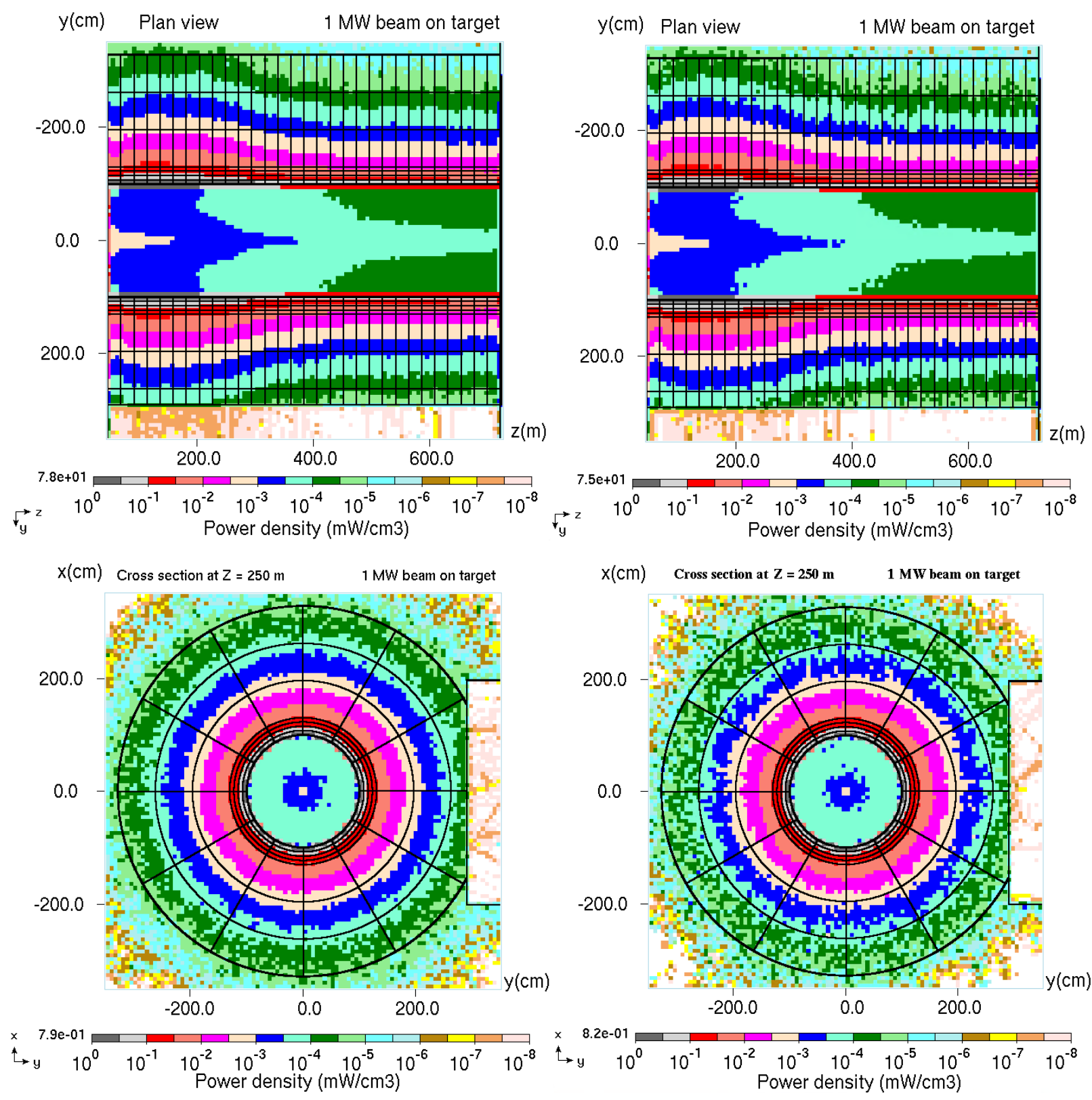

Fig. 16. Distributions of energy deposition in the decay pipe region calculated without (left) and with (right) the spatial-angular correlations in the incoming beam.

\section{References}

[1] I. Rakhno, Energy deposition and radiological studies for PIP-I+ with MARS15 code, Beams-doc-6488, Fermilab (2018).

[2] N.V. Mokhov and C.C. James, "The MARS code system User's Guide, Version 15 (2019)", Fermilab-FN-1058-APC (2017); https://mars.fnal.gov.

[3] N. Mokhov, private communication, Fermilab, 2020. 
[4] K. Yonehara, private communication, Fermilab, 2020.

[5] K. Ammigan, private communication, Fermilab, 2019.

[6] Y. He, private communication, Fermilab, 2019.

[7] I. Rakhno, Energy deposition and radiological studies with MARS15 code for NuMI-AIP project, Beams-doc-8389-v2, Fermilab (2020) 